Bull. Austral. Math. Soc.

22D05, 22D40

VOL. 65 (2002) [1-8]

\title{
ARC COMPONENTS IN LOCALLY COMPACT GROUPS ARE BOREL SETS
}

\author{
Karl Heinrich Hofmann
}

\begin{abstract}
Are the arc components in a locally compact group Borel subsets? An affirmative answer is provided for locally compact groups satisfying the First Axiom of Countability. For general locally compact groups the question is reduced to compact connected Abelian groups. In certain models of set theory the answer is negative.
\end{abstract}

\section{INTRODUCTION}

Recall that a Borel subset of a topological space is a member of the smallest sigmaalgebra of sets containing all open sets. We prove the following piece of information on locally compact groups.

THEOREM 1. In a first countable locally compact group the arc components are Borel subsets.

This is a partial answer to a question raised by Alexander Bendikov and Laurent Saloff-Coste in the context of [1]. For their purposes, the information on first countable locally compact groups suffices. Kunen and Starbird constructed a metric continuum in which some arc components ([6]), and Le Donne [7] used this to build one in which all arc components fail to be Borel subsets. The former authors asked whether a topological group on a continuum could have arc components failing to be Borel subsets. Theorem 1 above gives a negative answer.

Since the arc component $G_{a}$ of the identity in a topological group $G$ is contained in the identity component $G_{0}$ and $G_{0}$ is a closed subset, Theorem 1 deals in fact with locally compact connected groups. A locally compact connected group has maximal compact subgroups which are connected and any two of them are conjugate $[5,8]$. Let

\footnotetext{
Received 15th January, 2001

This article was completed while the author was a Visiting Scholar at the Centre for Industrial and Applicable Mathematics of the University of South Australia on an Australian Research Countil (ARC) Grant.

I thank Jim Rogers for having directed my attention to [6] and [7] after I lectured in Laszlo Fuchs' seminar at Tulane University. Fuchs gave me invaluable advice on Shelah's paper [9]: I am grateful to Alexander Bendikov and Laurent Saloff-Coste for having alerted me the apparently open question on locally compact groups treated here. I thank Sid Morris and the Centre for Industrial and Applicable Mathematics at the University of South Australia for their generous hospitality.
}

Copyright Clearance Centre, Inc. Serial-fee code: 0004-9727/02 \$A2.00+0.00. 
$K$ denote one of them. Its commutator subgroup $K^{\prime}$ is closed. Our first result will state that $G_{a}$ is a Borel subset of $G$ if and only if $\left(K / K^{\prime}\right)_{a}$ is a Borel subset of $K / K^{\prime}$ (1.3 below). This will reduce the issue of the Borel subset nature of the arc components of locally compact groups entirely to the domain of compact connected Abelian groups.

A locally compact first countable connected group has a countable basis for its topology and permits a metric which is compatible with the topology and makes $G$ into a complete metric space. In other words, such a $G$ is a Polish space ([2]). A space is a Lusin space if it is a bijective continuous image of a Polish space. Our proof will rest on the following theorem $([2$, Section 6 , number 7 , Théorème 3$])$.

THEOREM 2. A subset of a Lusin space is a Borel subset if and only if it is a Lusin space.

It turns out that in the context of Polish locally compact groups Lusin sets come in handy.

The weight of a space is the smallest of the set of cardinals of bases for its topology. A compact space is metrisable if and only if its weight is $\leqslant \aleph_{0}$. The Continuum Hypothesis states that the next cardinal $\aleph_{1}$ following the first infinite cardinal $\aleph_{0}$ is the cardinality $2^{\aleph_{0}}$ of the continuum. The Generalized Continuum Hypothesis asserts this for any infinite cardinal in place of $\aleph_{0}$. We shall prove that there are models of Zermelo Fraenkel set theory in which the Axiom of Choice and the Generalized Continuum Hypothesis hold and in which the following statement is true.

THEOREM 3. There is a compact Abelian group whose weight is the cardinality of the continuum and in which the arc components fail to be Borel subsets.

Let us consider the following statement.

The Borel Set Proposition. In any locally compact group, the arc components are Borel subsets.

It remains an open question whether this statement in undecidable in Zermelo Fraenkel Set Theory with the Axiom of Choice. Our results support the suspicion that this question may have an affirmative answer.

\section{Reduction to compact Abelian groups}

By a Theorem of Iwasawa ([5]), taken together with what we know since Yamabe's contributions to Hilbert's Fifth Problem ([8]), we have the following result.

PROPOSITION 1.1. Let $G$ be a connected locally compact group. Then there is a natural number $n$ and a maximal compact subgroup $K$ and a subspace $E \subseteq G$ which is homeomorphic to $\mathbb{R}^{n}$ such that the map $(k, e) \mapsto k e: K \times E \rightarrow G$ is a homeomorphism. Moreover, all maximal compact subgroups are conjugate.

In particular, any maximal compact subgroup of $G$ is connected. 
Recall that the commutator subgroup $K^{\prime}$ of a compact connected group is closed (see [4, Theorem 9.2, p.440]).

Lemma 1.2. Assume that $X$ and $Y$ are topological spaces and that $y_{0} \in Y$ is such that $\left\{y_{0}\right\}$ is a Borel subset of $Y$. Then a subset $B \subseteq X$ is a Borel subset of $X$ if and only if $B \times Y$ is a Borel subset of $X \times Y$.

Proof: The projection $p: X \times Y \rightarrow X$ is continuous. Hence if $B$ is a Borel subset then $B \times Y=p^{-1}(B)$ is a Borel subset of $X \times Y$. The function $x \mapsto\left(x, y_{0}\right): X \rightarrow$ $X \times\left\{y_{0}\right\}$ is a homeomorphism, and thus $B$ is a Borel subset of $X$ if and only if $B \times\left\{y_{0}\right\}$ is a Borel subset of $X \times\left\{y_{0}\right\}$. Since $\left\{y_{0}\right\}$ is a Borel subset of $Y$, the set $X \times\left\{y_{0}\right\}$, being full inverse image of the Borel subset $\left\{y_{0}\right\}$ in $Y$ under the projection $X \times Y \rightarrow Y$, is a Borel subset in $X \times Y$. Hence $B$ is a Borel subset of $X$ if and only if $B \times\left\{y_{0}\right\}$ is a Borel subset of $X \times Y$. Now assume that $B \times Y$ is a Borel subset of $X \times Y$. Then $B \times\left\{y_{0}\right\}=(B \times Y) \cap\left(X \times\left\{y_{0}\right\}\right)$, as the intersection of two Borel subsets of $X \times Y$, is a Borel subset of $X \times Y$, and thus $B$ is a Borel subset of $X$.

TheOrem 1.3. (The Reduction Theorem.) Let $G$ be a locally compact group, and let $K$ be any maximal compact subgroup of $G_{0}$. Then the following statements are equivalent.

(i) The arc components of $G$ are Borel sets.

(ii) The arc component of the identity $\left(K / K^{\prime}\right)_{a}$ in the compact connected Abelian group $K / K^{\prime}$ is a Borel subset.

Proof: The arc components of $G$ are all homeomorphic to $G_{a}$. Thus (i) amounts to saying that $G_{a}$ is a Borel set. Since $G_{a} \subseteq G_{0}$ and $K \subseteq G_{0}$ we may assume that $G$ is connected.

By Proposition 1.1, the function $(k, e) \mapsto k e: K \times E \rightarrow G$ is a homeomorphism where $E$ is homeomorphic to $\mathbb{R}^{n}$. By [4, Theorem 9.39, p.469], there is a compact connected Abelian subgroup $A \subseteq K$ such that $\left(a, k^{\prime}\right) \mapsto a k^{\prime}: A \times K^{\prime} \rightarrow K$ is a homeomorphism. Thus

$$
\left(a, k^{\prime}, e\right) \mapsto a k^{\prime} e: A \times K^{\prime} \times E \rightarrow G
$$

is a homeomorphism. The subgroup $K^{\prime}$ is arcwise connected (see [4, Theorem 9.19, pp. 450-452 and Theorem 9.60, p.487]). Hence $K^{\prime} \times E$ is arcwise connected. Thus the arc component $G_{a}$ is homeomorphic to $A_{a} \times K^{\prime} \times E$. Hence by Lemma $1.2, G_{a}$ is a Borel subset of $G$ if and only if $A_{a} \times G^{\prime} \times E$ is a Borel subset of $A \times K^{\prime} \times E$ if and only if $A_{a}$ is a Borel subset of $A$.

Let us now consider a compact connected Abelian group $A$. The union of all circle subgroups is contained in a unique fully characteristic smallest closed subgroup $A_{\ell}$, 
called the locally connected component of $A$ (see [4, Definition 8.40, p.400]). We now refer to [4, Theorem 8.46, pp.404, 405], and summarise it as follows.

Lemma 1.4. Let $A$ be a compact connected Abelian group and assume that its locally connected component $A_{\ell}$ is metric. Then

(i) $A_{\ell}$ is a torus (that is, a product of circle groups).

(ii) There is a closed connected subgroup $H$ of $A$ such that $A \cong A_{\ell} \times H$. Moreover, exp: $\mathfrak{L}(H) \rightarrow H$ is injective.

Lemma 1.5. Let $A$ be a compact connected Abelian group and $T$ a torus subgroup (that is, a product of circle groups). Then the following conditions are equivalent.

(i) $A_{a}$ is a Borel subset of $A$.

(ii) $(A / T)_{a}$ is a Borel subset.

Proof: From [4, Theorem 8.78, p.426] we know that $A \cong T \times A / T$. Now $(T \times A / T)_{a}=T \times(A / T)_{a}$. The assertion now follows from Lemma 1.2.

\section{LUSIN SPACES}

We refer particularly to [2, Chapter 9]. A Polish space is a completely metrisable second countable space (see [2, Section 6 , number 1 , Definition 1]). This applies to $G$. We don't have to know exactly what a Lusin space is, but we shall use that concept. By Section 6, number 4, Definition 6, it is a metrisable space which is a bijective continuous image of a zero dimensional Polish space. The following is relevant.

LEMMA 2.1. $\mathbb{R}$ is a Lusin space.

Proof: It is shown in Bourbaki, [2, Section 6, no 4, Lemme 2], that a metric space which is a countable disjoint union of Lusin spaces is a Lusin space. Now $\mathbb{R}$ is a countable disjoint union of singleton sets containing rational points and the set of irrationals. The latter is a zero dimensional Polish space, hence is a Lusin space. Therefore $\mathbb{R}$ is a Lusin space.

Since a countable product of zero dimensional Polish space is a zero dimensional Polish space we conclude the following.

LEMmA 2.2. A countable product of Lusin spaces is a Lusin space.

Hence $\mathbb{R}^{n}, n \in \mathbb{N}$ and $\mathbb{R}^{\mathbb{N}}$ are Lusin spaces.

Lemma 2.3. If $A$ is a metrisable compact Abelian group, then $\mathfrak{L}(A)$ is a Lusin space.

Proof: As a topological vector space, $\mathfrak{L}(A)$ is isomorphic to one of the vector spaces $\mathbb{R}^{n}, n \in \mathbb{N}$, or to $\mathbb{R}^{\mathbb{N}}$. (See $[4$, pp. $\left.334-342,355,356]\right), \mathfrak{L}(A) \cong \operatorname{Hom}(\widehat{A}, \mathbb{R}) \cong$ $\mathbb{R}^{F}$ where $F$ is a maximal free subset of the countable character group $\hat{A}$.) 
PRoposition 2.4. Every bijective continuous image of a Polish space is a Lusin space.

Proof: See [2, Section 6, number 4, Proposition 12].

Corollary 2.5. The arc component $A_{a}$ of a compact connected metric Abelian group $A$ is a Borel subset.

Proof: By Lemma 1.4, $A$ is isomorphic to the direct product of the torus $A_{\ell}$ and a metric compact subgroup $H$ for which exp: $\mathfrak{L}(H) \rightarrow H$ is injective. Now $\mathfrak{L}(H)$ is a Lusin space by Lemma 2.3. But then $H_{a}=\exp _{H} \mathfrak{L}(H)$ is the bijective image of the Lusin space $\mathfrak{L}(H)$ and thus is a Lusin space by Proposition 2.4. Then by Theorem 2 in the Introduction $\left(A / A_{\ell}\right)_{a} \cong H_{a}$ is a Borel subset of $A / A_{\ell}$. Hence $A_{a}$ is a Borel subset of $A$ by Lemma 1.5 .

Now we are ready for the second main result.

Theorem 2.6. (Arc components and Borel sets.) Let $G$ be a locally compact group and $K$ a maximal compact subgroup of the identity component $G_{0}$. Assume that the compact connected Abelian group $K / K^{\prime}$ is metric. Then the arc components of $G$ are Borel subsets.

ProOF: By the Reduction Theorem 1.3, the assertion is true if and only if the arc component $\left(K / K^{\prime}\right)_{a}$ is a Borel subset of $K / K^{\prime}$. Since $K / K^{\prime}$ is assumed to be metric, the assertion follows from Lemma 2.5.

Of course there are numerous sufficient conditions which imply the hypothesis that $K / K^{\prime}$ is metric.

PROPOSITION 2.7. For a locally compact group, each of the following conditions implies the next.

(i) $G$ is metric.

(ii) $G_{0}$ is metric.

(iii) The maximal compact connected normal subgroup $K$ of $G_{0}$ is metric.

(iv) The identity component $Z_{0}(K)$ of the centre of $K$ is metric.

(v) The compact connected Abelian group $K / K^{\prime}$ is metric.

Proof: Since $Z_{0}(K) \subseteq K \subseteq G_{0} \subseteq G$, the first three implications are obvious. For (iv) $\Rightarrow(v)$ we refer to $[4,9.23$ p.456, or 9.24, p.458] and conclude thăt $Z_{0}(K) /\left(Z_{0}(K) \cap K^{\prime}\right) \cong K / K^{\prime}$. Thus $K / K^{\prime}$ is a homomorphic image of $Z_{0}(K)$ and the implication follows.

In particular, Theorem 1 of the Introduction is implied by Theorem 2.6 and Propositiion 2.7. 


\section{THE REVERSE OF THE COIN}

The Reduction Theorem 1.3 shows that the question whether arc components are Borel sets reduces to the same question for compact Abelian groups. For these we know that in the metric case the answer is yes. For a compact group $G$ to be metric means that the weight $w(G)$ is at most $\aleph_{0}$ (see [4, Exercise EA4.3, pp.763, 764]).

We now consider the negation of the Borel Set Proposition of the Introduction.

The Anti-Borel Set Proposition. There exists a compact Abelian group $G$ of weight $w(G)=\aleph_{1}$ such that $G_{a}$ is not a Borel set.

We shall argue that there is a model of set theory in which the generalised continuum hypothesis and the Axiom of Choice hold in which the Anti-Borel Set Proposition holds.

We do not know whether in a constructible universe the arc components of all locally compact groups are Borel sets. A proof of this fact would show that the Borel Set Proposition (of the Introduction) is undecidable in Zermelo Fraenkel Choice.

It appears to be very hard to determine that a given subset of a topological space is not a Borel subset $([\mathbf{1 0}])$. In a compact group there is a classical trick that provides a sufficient condition.

PROPOSITION 3.1. Let $C$ be a subgroup of a compact group $G$ such that the set $G / C$ of cosets $g C$ is countably infinite. Then $C$ is not Haar measurable and thus is not a Borel subset.

Proof: Let $m$ be normalised Haar measure on $G$ and suppose that $C$ is Haar measurable. Let $g_{1}, g_{2}, \ldots$ be a sequence of elements such that $\left\{g_{n} C: n \in \mathbb{N}\right\}$ is an enumeration of $G / C$. Then for each natural number $N$, using the invariance of $m$, we have

$$
N \cdot m(C)=\sum_{n=1}^{N} m\left(g_{n} C\right)=m\left(g_{1} C \cup \cdots \cup g_{N} C\right) \leqslant m(G)=1
$$

Hence $m(C) \leqslant 1 / N$ for all $N=1,2, \ldots$ and thus $m(C)=0$. But then $1=m(G)=$ $m\left(\bigcup_{n \in \mathbb{N}} g_{n} C\right)=\sum_{n \in \mathbb{N}} m\left(g_{n} C\right)=0$. This contradiction shows that our supposition is false.

We now cite the following proposition from [4, Theorem 8.30, p.389].

Proposition 3.2. Let $G$ be a compact $A$ belian group and $A$ its character group. Then the (abstract) factor group $G / G_{a}$ is isomorphic to $\operatorname{Ext}(A, \mathbb{Z})$.

Thus Propositions 3.1 and 3.2 motivate us to formulate the following statement.

The Countability Proposition. There is an Abelian group $A$ such that $\operatorname{Ext}(A, \mathbb{Z})$ is countably infinite. 
By Propositions 3.2 and 3.1, the Countability Proposition implies the Anti-Borel Set Proposition.

The author is indebted to Laszlo Fuchs who reported the following fact.

The Countability Proposition is undecidable in Zermelo Fraenkel Choice.

Indeed, first of all, if $\mathrm{A}$ is countable, but not free, then $\operatorname{Ext}(A, \mathbb{Z})$ is always uncountable. For higher cardinalities, in Gödel's constructible universe it is either 0 or its torsion-free part is uncountable (see for example, [3, Chapter XII, in particular, Corollary 2.5]).

On the other hand Shelah [9] has shown that even if the Generalized Continuum Hypothesis is assumed, for every countable divisible group $D$ there is a model of set theory in which there is an Abelian group $A$ of cardinality $\aleph_{1}$ such that $\operatorname{Ext}(A, \mathbb{Z}) \cong D$.

Shelah's proof starts with any model of Zermelo Fraenkel Choice and Generalized Continuum Hypothesis and he shows by forcing that, for a given countably infinite divisible group $D$, it has an extension in which, for a suitable $A$, the group $\operatorname{Ext}(A, \mathbb{Z})$ is isomorphic to $D$.

In particular, for the additive group $\mathbb{Q}$ of rational numbers there is a model of set theory in which there is a compact Abelian group $G$ of weight $\aleph_{1}=2^{\aleph_{0}}$ such that the arc component factor group $G / G_{a}$ is algebraically isomorphic to $\mathbb{Q}$; thus the arc components of $G$ fail to be Borel subsets.

This produces Theorem 3 in the Introduction.

\section{REFERENCES}

[1] A. Bendikov and L. Saloff-Coste, 'On the absolute continuity of Gaussian mesures on locally compact groups', (TEX-script May 23, 2000).

[2] N. Bourbaki, Topologie générale (Hermann, Paris, 1974).

[3] P.C. Eklof and A.H. Mekler, Almost free modules: set theoretic methods, North-Holland Mathematical Library 46 (North Holland, Amsterdam, 1990).

[4] K.H. Hofmann and S.A. Morris, The structure of compact groups, de Gruyter Studies in Mathematics 25 (Walter de Gruyter, Berlin, 1998).

[5] K. Iwasawa, 'On some types of topological groups', Ann. of Math. 50 (1949), 507-558.

[6] K. Kunen and M. Starbird, 'Arc components in metric continua', Topology Appl. 14 (1982), 167-170.

[7] A. Le Donne, 'Arc components in metric continua', Topology Appl. 22 (1986), 83-84.

[8] D. Montgomery and L. Zippin, Topological transformation groups (Interscience Publishers, New York, 1955).

[9] S. Shelah, 'The consistency of $\operatorname{Ext}(G, \mathbb{Z})=\mathbb{Q}$ ', Israel J. Math. 39 (1981), 74-82.

[10] S. Shelah, 'A space with only Borel subsets', Period. Math. Hungar. 40 (2000), 81-84. 
Fachbereich Mathematik

Technische Universität Darmstadt

Schlossgartenstr. 7

D-64289 Darmstadt

Germany

e-mail: hofmann@mathematik.tu-darmstadt.de 